\title{
Téoros
}

Revue de recherche en tourisme

\section{Le réseau piétonnier intérieur : une place importante dans l'industrie touristique de Montréal}

\section{Sandrine Brillet}

Volume 10, numéro 3, novembre 1991

Destination Montréal : d'hier à demain

URI : https://id.erudit.org/iderudit/1079196ar

DOI : https://doi.org/10.7202/1079196ar

Aller au sommaire du numéro

Éditeur(s)

Université du Québec à Montréal

ISSN

0712-8657 (imprimé)

1923-2705 (numérique)

Découvrir la revue

Citer cet article

Brillet, S. (1991). Le réseau piétonnier intérieur : une place importante dans

l'industrie touristique de Montréal. Téoros, 10(3), 9-12.

https://doi.org/10.7202/1079196ar d'utilisation que vous pouvez consulter en ligne. 
A la veille de la commémoration du 350 ème anniversaire de Montréal, le réseau piétonnier intérieur du centre-ville de Montréal ou Montréal souterrain se veut définitivement tourné vers une politique d'identification et de valorisation touristique. Il n'est pas, à ce jour, un guide touristique, un livre d'histoire ou un article de presse sur Montréal qui oublient de citer et de décrire la ville souterraine comme un phénomène unique au monde par sa tailleet son ancienneté. L'Office des congrès et du tourisme du Grand Montréal écrit dans son guide touristique: $\mathrm{La}$ Cité piétonne ne cesse de s'agrandir pour offrir aux Montrêalais et aux visiteurs un système de transport et de circulation unique par son ampleur et ses qualités; véritable percée qui fait de Montréal la première ville du XXIe siècles,

Ville parallèle, ville dans la ville, prototype de la ville du futur, le Montréal souterrain a doublé sa superficie entre 1984 et 1989. Désormais, 25 kilomètres de corridors desservent 2.9 millions de mètres carrés de plancher. Dans deux ans, le Montréal souterrain donnera accès, grâce à 30 kilomètres de corridors, à $80 \%$ de tous les bureaux du centre des affaires, 1700 établissements commerciaux, 4230 chambres d'hôtels, 1615 logements, etc. Impossible donc de parler du centre-ville de Montréal sans parler de cette ville dans la ville qui attire chaqueanne des milliers de touristes. Avec un potentiel commercial et architectural considérable, le Montréal souterrain s"affirme aujourd'hui comme le pôle d'attraction touristique du centre-ville. Il occupe une place prépondérante dans la vie économique de la ville et participe à la promotion de son image touristique. Il appartient pourtant aux professionnels du tourisme montréalais de mettre en valeur ces richesses touristiques et de promouvoir l'originalité de son image sous l'égide d'une politique motivée et concertée. L'année 1992 devrait permettre de définir une politique concrète d'aménagement et d'attirer l'attention des touristes sur un produit original, malgré une concurrence nord-américaine tres active.

Madame Sandrine Brillet est étudiante à la maítrise enamenagement al'Universite de Paris I, Panthiton. Sorbonne.

\section{Le plus ancien réseau piétonnier intérieur d'Amérique du Nord}

Le réseau piéton intérieur montréalais est le plus vaste et le plus ancien d'Amérique du Nord. C'est avec la construction de la Place Ville-Marie, entre 1958 et 1962 , qu'il est né. Ce complexe multifonctionnel marque le début des premières galeries commerciales souterraines et l'avènement d'un nouvel essordu centre-ville. L'ouverture du métro, en 1966, est la seconde étape importante du développement đu réseau piéton intérieur.

Dans les anneses 1960 et 1970 , les promoteurs et les gestionnaires d'équipement comprennent très vite l'importance stratégique de la création de circulations. piétonnes directement reliếes au métro. Aussi, le réseau piéton intérieur dessert sans attendre les magasins à rayons Eaton et La Baie, les deux gares de chemins de fer et les grands hôtels du centre-ville. Il a, de ce fait, été conçu comme une extension du métro. Il va sans dire que, sans lui, l'achalandage quotidien des espaces commerciaux intérieurs n'aurait jamais connu pareille intensité et succès. Ainsi, au fil des années, le réseau piétonnierintérieurs'étend de façon régulière. Mais il n'obéit à aucune planification d'ensemble. En fait, il évolue au gré des promoteurs immobiliers qui privilégient, quel qu'en soit le prix, la connexion au réseau (construction et gestion). Depuis quelques années, les projets de construction des grands édifices ou équipements du centre-ville dépendent en priorité de cette connexion, même lorsque la fonction commerciale y joue un rôle mineur.

\section{Une étroite relation entre le climat et la revitalisation commerciale du centre-ville}

Quoiqu'il en soit, le Montréal souterrain est né d'une volonté de s'adapter aux contraintes climatiques auxquelles est exposée la ville d'une saison à une autre, de créer un réseau de circulation piétonnière protégée, de rentabiliser l'espace souterrain nécessaire aux fondations des gratte-ciels, enfin et surtout, de sauvegarder la vitalité commerciale du centre-ville. Le Montréal souterrain s'explique d'abord et avant tout par le développement de l'activité com- merciale en centre-ville explique Michel Boisvert, économiste et professeur à l'Institut d'urbanisme de l'Université de Montréal.

En fait, le réseau piétonnier intếrieur de Montréal s'inscrit dans une problématique d'ensemble des réseaux piétonniers prot'égés d'Amérique du Nord. Comme à Toronto, Calgary, Minneapolis ou Edmonton, le réseau montréalais se décritcomme un urbanisme destiné au confort des déplacements piétonniers en centre-ville, comme un axe *obliges et prestigieux de développement économique et comme un attrait touristique au coeur d'un grand centre urbain.

\section{Un découpage sectoriel en sept zones d'intérêt touristique}

Comme l'illustre le schếma 1, le Montréal souterrain peut être divisé en sept secteurs piétons:

Atwater, le secteur le plus excentrét:

- Berri-UQAM, an coeur du squartier latins:

- Bonaventure, le secteur piétonnier originel;

- McGill, le secteur le plus achalandé;

- Peel, le secteur piétonnier le plus rếcent:

- Place-des-Arts/Place d'Armes, l'ensemble «culturel et gouvernementals:

- Square Victoria, au coeur du centre des affaires.

La Ville de Montréal et les professionnels du tourisme montréalaisentendent, par cette politique de découpage sectoriel, mettre en évidence le caractère touristique original dechaquesecteur. Ainsi, le secteur McGill, plus commercial dans ses fonctions, se différencie-t-ildusecteurPlace-des-Arts/Place d'Armes, plus culturel et artistique. D'ailleurs, la Ville de Toronto a adopté, la première, ce principe en divisant l'Underground Pedestrian Mall System en huit zones d'intérêt touristique. Cela facilite de façon certaine la découverte touristique du réseau piétonnier et permet de créer plusieurs pôles d'attraction pour le touriste. 
En fait, Montréal a pris du retard dans ses efforts d'identification et de valorisation touristique. Il est vrai que la politique de Toronto s'est voulue, dès le début (et au contraire de Montréal), en faveur d'un contrôle puissant du secteur public et d' une planification détaillée du réseau piếtonnier intêrieur. C'est aujourd' hui au tour de la Ville de Montréal de prendre officiellement conscience de l'important potentiel touristique du Montreal souterrain et de définir une politique touristique appropriée.

\section{Une concentration étonnante de l'infrastructure touristique de la ville}

Le Montréal souterrain constitue une concentration du parc hôtelier montréalais de luxe ainsi que des principaux equipements de congrès, de loisirs et de spectacles de la ville. Ainsi, cette concentration apporte la démonstration d'un potentiel touristique reposant sur la réalité et la densité d"un tissu d'équipements d'agrément varié et important.

Le parc hờtelier relié au réseau piếtonnier intérieur représente l'élément le plus significatif de cette infrastructure. En effet, le réseau piétonnier intérieur s"est donné, au fil des années, le privilè̀ge de relier sept des plus grands et des plus prestigieux hotels du centre-ville: le Reine Elizabeth, Le Grand Hôtel, le Château Champlain, le Bonaventure Hilion, le Mẹridien MontrGal, 1'Hôtel des Gouverneurs - Place Dupuis et l'Intercontinental Montréal. Aujour'd hui, le parc hôtelier directement relié au réseau piétonnier intérieur représente, en nombre de chambres, environ $40 \%$ du pare hôtelier du centre-ville. Une analyse de contenu des dépliants publicitaires des hôtels permet facilement d'alfirmer que le lien avec le réseau piótonnier intérieur représente une stratégie de vente percutante pour les gestionnaires et une image de prestige aupres des différentes clientèles.

Il est également un argumente vente pour les gestionnaires et organisateurs de congres. Le Palais des congres, avec 31 salles de réunions, soit environ $14000 \mathrm{~m}^{2}$ de surface de plancher, dispose de la plus grande capacite d'accueil de la ville. Il est relié au secteur Place-des-Arts/Place d'Armes. Avec le secteur Bonaventure, ils constituent un potentiel d'accueil et d'expositions très important, à savoir plus de $30000 \mathrm{~m}^{2}$ de surface d'expositionset de salles de réunions.

\section{TABLEAU 1}

Activités reliếes au réseau piétonnier intêrieur de Montrèal (centre-ville incluant la station de métro Atwater)

\begin{tabular}{|c|c|c|c|}
\hline & 1984 & 1989 & $1992 *$ \\
\hline Palais des congrès & 1 & 1 & 1 \\
\hline Musée d'Art Contemporain & - & - & 1 \\
\hline UQAM & 1 & 1 & 1 \\
\hline Gares de chemin de fer & 2 & 2 & 2 \\
\hline Gare d'autobus interurbains & 1 & 1 & 1 \\
\hline Grands hôtels & 7 & 6 & 9 \\
\hline Nombre de chambres & 4968 & 3900 & 4617 \\
\hline Habitations & 1099 & 1250 & 1615 \\
\hline Grands magasins à rayons & 3 & 3 & 2 \\
\hline Boutiques & 800 & 1150 & 1600 \\
\hline Restaurants-bars & 78 & 149 & 210 \\
\hline Cinemas/theâtres & 26 & 27 & 34 \\
\hline Succursales de banques & 36 & 40 & 45 \\
\hline Places de stationtement souterrain & - & 11200 & - \\
\hline Total des établissements commerciaux & 940 & 1200 & 1700 \\
\hline Total de l'espace commercial (m2) & 543800 & 613500 & 695500 \\
\hline Espaces de bureaux (m2) & 1603800 & 1758650 & 2285500 \\
\hline Nombre de travailleurs & 96000 & 100000 & 170000 \\
\hline \multirow{2}{*}{$\begin{array}{l}\text { Nombre de persontues qui empruntent } \\
\text { chaque jour le réseau piétonnier } \\
\text { intérieur }\end{array}$} & & & \\
\hline & 300000 & 300000 & 330000 \\
\hline * Previsions & & & \\
\hline
\end{tabular}

Source; SHDU (Ville de Montréal).

Par ailleurs, les scènes de spectacle montrúalaises jouent aussi un rôle important dans le paysage social et touristique du Montréal souterrain. La Place-des-Arts domine bien entendu l'activité culturelle et artistique du Montréal souterrain. Elle est le plus important centre intégré des arts de la scène à Montrüal. Elle dispose de quatre salles de spectacle pouvant contenir un total de plus de 5000 spectateurs. De plus, le gouvernement du Québec a rendu officiels trois nouveaux projets d'agrandissement et de valorisation du site, en plus de la construction du Musée d'Art Contemporain:

- la construction d'un nouveau thêatre de 400 places;

- l'agrandissement de la salle Wilfrid= Pelletier;

- la construction d'un nouveau parcautos en-dessous dú nouvel édifice du Musée d'Art Contemporain.

Ainsi, la Place-des-Arts s'affirme comme le centre culturel par excellence de la ville, mais aussi comme une place publique qui regroupe, au sein du Montréal souterrain, les principaux éléments culturels de la ville.

Parallèlement, le Centre Infotouriste se veut un organisme d"accueil et d'information auprés des touristes de la ville. $\mathrm{Or}_{4}$ il jouera bientôt un rôle plus étroit avec le Montréal souterrain, puisque le Centre, situé Square
Dorchester, sera probablement relie par corridor souterrain au secteur Peel.

\section{Les attraits touristiques du Montréal souterrain: un symbole architectural et culturel à définir}

En plus des equipements d'agrếment et des services touristiques auxquels il donne accès, le Montréal souterrain offre un nombre important d'atraits qui renforcent son image et son activité touristiques. Ainsi, les places ou complexes commerciaux sont les principaux pôles d'attaction. La qualité et l'originalité de leur décoration intérieure, leurs qualités d'intégration avec l'extérieur (Place Montréal Trust, Centre Eaton, ConplexeDesjardins, la Place-des-Arts,etc) et le raffinement des boutiques égayent et adoucissent un paysage commercial résolument agressif. De plus, il est toujours laissé à l'intérieur une place centrale publique autour de laquelle architectes et designers ont recrée un jardin ou un oasis. Ainsi, plantes, fontaines, cascades d'eau, sculptures géantes transforment-ils ces espaces fermés en de véritables el dorados pour le plaisir des piétons et des touristes. Aussi, que les visiteurs recherchent l'animation du centre commercial, la quiếtude du jardin artificiel ou le symboled'une architecture intégrée contemporaine, les complexes s'affirment comme de véritables pôles d'attraction touristique. S'ajoutentếgalement à eux un certain nom- 


\section{SCHEMA 1}

Le réseau piétonnier intérieur de Montréal en 1991 et les immeubles du centre-ville qui y sont reliés
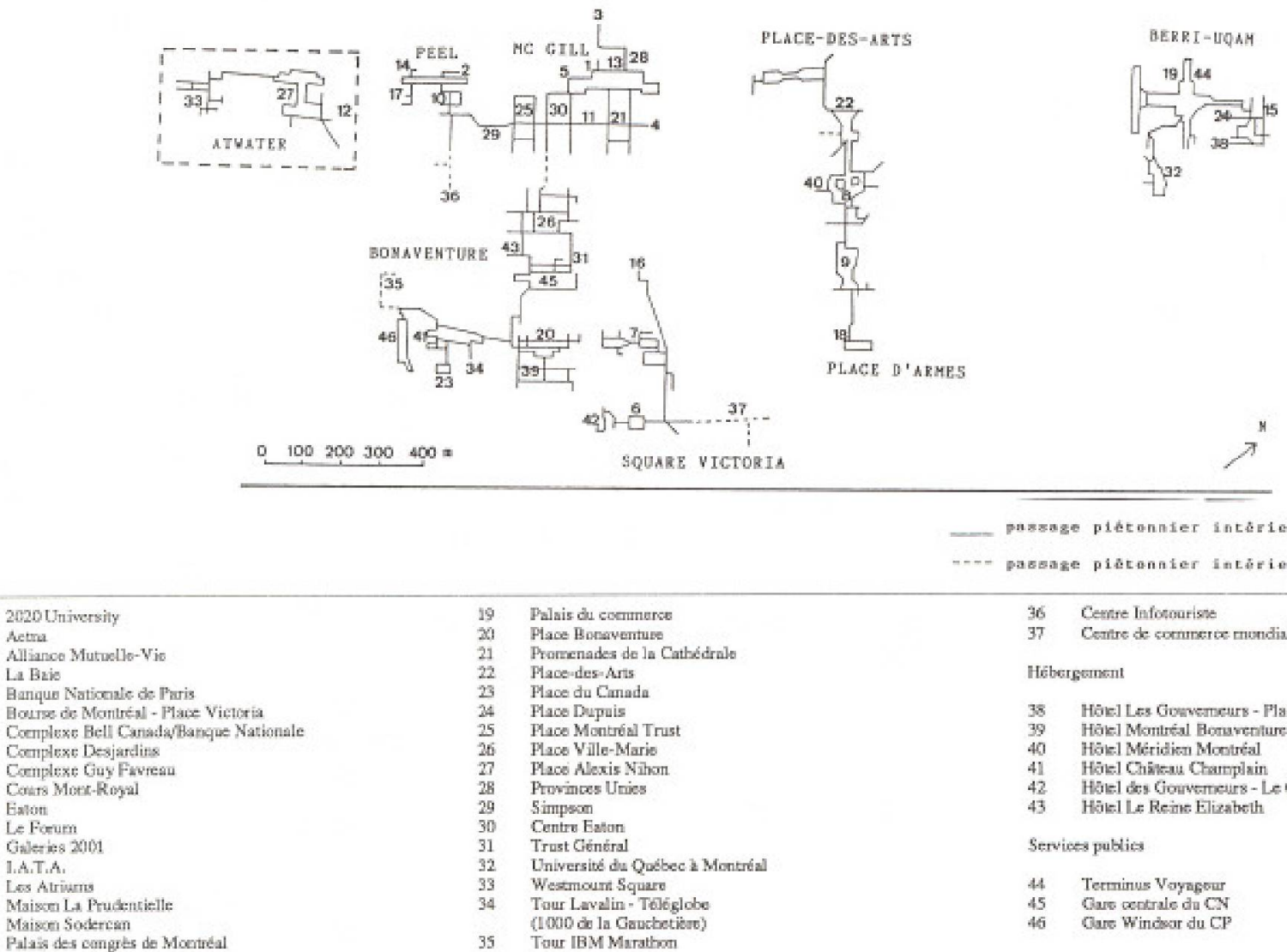

bre d'attraits propres à l'histoire et l'economie de Montréal. Ainsi, les tours de bureaux, le Muséd ${ }^{+}$Art Contemporain dans le centre culturel, l'expétience architecturale réussie etoriginale de l'Universitédu Québec à Montúval, les gares de chemins de fer (Gares Centrale et Windsor'), les magasinsEatonet La Baie, l'Eglise Christ Church, seul édifice religieux re lié au Montréal souterrain, etc, sont autant d'attraits incontoumables pour le touriste. Aussi, le potentiel touristique du Montréal souterrain se veut varié, important, parfois chargé d'histoire etouvert à l'accueil des touristes. Pourtant, le rôle des intervenants dans l'identification touristique et la valorisation du Montrcal souterrain se veut encore trop discret. Cing fonctions majeures devraient permettre prochainement d'aboutir à une politique touristique active et efficace.

La planification et le contrôle de I’extension du Montréal souterrain

Le secteur public, par l'intermédiaire du Service de l'habitation et du développement urbain (SHDU, Ville de Montréal), entend réaffirmer sa responsabilité de contrôle face à l'engouement immobilier des promoteurs prives. Il própare une planification détaillée du réseau piétonnier intérieut afin de parvenir à une meilleure intégration avec le cadre bâti extérieur et d'appliquer des règles strictes d'aménagement physique.

\section{La gestion et la valorisation de la qualité ambiante dans le Montréal souterrain}

Différents projets importants, tels que la signalisation touristique du réseau piétonnier intérieur (présentement à l'étude), mettent en évidence un souci d'identifier le Montréal souterrain, de l'intégrer à la rue, de promouvoir son utilisation et celle de ses composantes, d'informer le public et l'orienter à l'intếrieur comme à l'extérieur, enfind ànimer le réseau. Cependant, il faut espérer que la multiplicité des projets en cours ne parviendra pas à créer une pollution visuelle ou une surcharge de nobilier urbain. Seule la concertation des projets et des intervenants réussira à valoriser le
Montréal souterrain sans détruire son caractère propre et agréable à vivre.

\section{Des outils d'information et de découverte touristique}

$\mathrm{L}^{4}$ Office des congres et du tourisme du Grand Montréal devrait élaborer un guide touristique du Montréal souterrain (il faut rappeler que Toronto possède son propre guide du réseau piétonnier intérieur depuis deux ans déjà). De cette façon, le touriste pourrait faire une meilleure utilisation dece réseau et du réseau commercial. Le guide devrait également servir de support historique, architectural et artistique. Ce document est indispensable à la promotion du Montréal souterrain. De mẹme, il apparait urgent de réaliser des cartes de visualisation et d'orientation schématique du Montréal souterrain: le système de représentation axonométrique serait d'ailleurs le meilleur moyen d'identifier le Montréal souterrain dans l'ensemble de son architecture intérieure et extérieuré. Il serait également plus efficace et évocateur pour le touriste. 
TABLEAU 2

Montréal souterrain: appréciation des touristes

Critique favorable Critique défaworable

L'originalitế du produit touristique.

La structure architecturale.

La dynamique culturelle, ou la rencontre

avec un mode de vie citadine.

Le lieu de rencontre, la place publique.

La taille et la quasi-oontinuité du réseau.

Le confort et la sécurité des déplacements.

L'aménagement intérieur et la décorationt.

L'animation culturelle et artistique.

Les puits de lumière naturelle.

Les façades vitrées sur rue.

La prupreté des corridors.

La hauteur des corridors souterrains.

Source; BOIVIN, D., Montréal souterrain: étude du réseau piétonnier protégé du centreville, 1989. GOUDREAU, J., Guide touristique â Montréal.

Une politique concertee de promotion touristique

La politique touristique de Montréal se veut très attaché à la variété et la qualité des événements culturels, historiquesousportifs qui s'y déroulent. Le Montréal souterrain y trouve de plus en plus sa place. Ainsi, la semaine montréalaise de sensibilisation au tourismẹ et le 25 ème anniversaire du métro montré́alais (automne 1966 - automne 1991), partenaire obligé du réseau piétonnier intérieur, accordent-ils au Montréal souterrain l'occasion de mieux se faire connâtre du grand public et des touristes. D'autre part, I'OCTGM a lancé au début de l'annê une expérience tout à fait originale et innovatrice aupress d'un groupe améri= cain de familiarisation: un rallye dans la ville souterraine. Cette initiative, couronnée semble-t-il d'un vif succès, est bien entendu à encourager et à développer auprès du grand public. D'autres outils médiatiques, tels que les vidéocassettes, offrent Également l'opportunité de se familiariser au réseau piétonnier intérieur et de découvrir ses richesses touristiques.

\section{Un produit touristique commercial et culturel}

Ilest difficile d'apprécier avec précision le nombre de touristes qui parcourent chaque année le Montréal souterrain. Le Groupe Trizec, promoteur de la Place Ville-Marie, a établi une enquête au début de l'année 1991 sur l'achalandage naturel de la place et de ses terrasses. Or, l'enquête révèle que sur les 300000 personnes qui traverseraient chaque semaine la Place Ville-Marie, $19 \%$ seraient des touristes, soit plus de 50000 visiteurs par semaine. Ce chiffre met en
Le manque de signalisation touristique. La longueur de certains corridors souterrains. Le manque d'affichage sut les murs des corridors souterrains.

L'éclairage souterrain.

Les odeurs de cuisine à l'intérieur des complexes.

Le bruit.

évidence la réalité d'une clientèle dont le molif de déplacement dans lẹ réseau piétonnier intérieur n'est autre que le tourisme.

En fait, I'ểlếment commercial influence de façon significative l'atractivité touristique du Montréal souterrain, surtout auprès des clientẻles américaine et japonaise. La clientèle européenne se veut, quant à elle, plutot attire par le symbole architectural et la waçon de vivres dans le Montréal souterrain.

Les agences de tourisme montréalaises vendent des tours guidés de la ville souterraine. Selon elles, le produit est très couru en hiver, mais il est souvent combine avec un autre thème, par exemple, le combiné «Vieux-Montréal et Montréal souterrain\%, soit le Montréald'hier etd'aujourd'hui. De facon générale, les parcours privilégient les axes Places-des-Arts/Placed'Armes, Cours Mont-Royal/La Baie et Place Ville-Marie/ Gare Windsor. Mais le produit gagne actuellement, selon les agences de tourisme, en qualité et variété de présentation. It s'enrichit et se veut plus culturel que comnercial. Les Montrealistes (entreprise de tours guidés) jouent, par exemple sur la thématisation des visites en rapport avec l'actualité culturelle de la ville. Ainsi, l'agence prépare pour' le printemps prochain un projet de visite d'une demi-journé dans la ville souterraine dans laquelle serait incluse, soit une visite des arrières-scênes de spectacle de la salle Wilfrid-Pelletier à l'occasion d'un événement-spectacle soit une visite dans le nouveau Musée d'Art Contemporain à l'occasion d'une exposition. Pour les Montrealistes, c'est le contenu culturel et social du Montréal souter- rain qui fait I'originalité du produit. Il représente un vécu animé par une dynamique culturelle tartistique importante. II n'est pas une structure abstraite ou une simple armature architecturale. Il vit. Le découvrir et le connaitre, c'est le sentir vivre ou le vivre.

\section{Un effort d'aménagement et de valorisation touristique}

Al'heureactuelle, lesccteurpublicetlesprofessionnels du tourisme unissent leursefforts pour palier un certain nombre de problemes dans le fonctionnement du Montréal souterrain, tels que la signalisation touristique, la decoration intérieure ou plus exactement l'interaction cclairage/couleur, laqualité del'airet lesheures d'ouverture. Tous les intervenants souhaitent ainsi parvenir à une identification d'ensemble du Montréal souterrain, et ce, grâce à une signalisation touristique standardisce et efficace et une politique de confort et d'homogénéisation dans la qualité ambiante.

De plus, le secteur public entend gérer le développenent du réseau piétonnier intérieur vers un réseau continu. Ainsi, une connexion importante sera bientôt ouverte entre le Centre Eaton et la Place Ville-Marie. Il s'agit d'un Évencment majeur pour le Montréal souterrain.

\section{Une image de marque et un rôle dans l'économie du Montréal souterrain}

Ainsi, à la veille d'un événement touristique aussi important que le 350ème anniversaire de la Ville de Montréal, il semble que tous les intervenants soient prets à s'entendre sur la nécessité de la mise en valeur et de la promotion du Montréal souterrain. Eneffet, pourl' industrie touristique montróalaise, 1992 est un véhicule promotionnel considérable et d'une portée intemationale. Des milliers de touristes sont attendus à Montráal pour l'occasion. II revient donc, désormais, aux professionnels dutourisme de profiter de l'évếnement pour appliquer une politique concrète de mise en valeur archilecturale, commerciale, touristique, culturelle et artistique du Montreal souterrain, atind'assurer son développement harmonieux et son success economique.

Tels sont le sens et la ré́ussite du produit touristique et culturel, tel doit être aussi son avenir. Les conditions d'aménagement du Montrếal souterrain détermineront la vitalité du réseau commercial et le succès du produit touristique. Le Montréal souterrain défend aujourd' hui une image de marque. Il détient, avec le tourisme, l'opportunitéde renforcersonrôlécononnique. 